\title{
Low Social Support and Risk for Depression in People With Type 2 Diabetes Mellitus: A Systematic Review and Meta-analysis
}

\author{
Akhmad Azmiardi ${ }^{1}{ }^{1}$, Bhisma Murti ${ }^{1}$, Ratih Puspita Febrinasari ${ }^{3}$, Didik Gunawan Tamtomo ${ }^{1}$ \\ ${ }^{1}$ Doctoral Program on Public Health, Universitas Sebelas Maret, Surakarta, Indonesia; ${ }^{2}$ Department of Public Health, Universitas Veteran Bangun \\ Nusantara, Sukoharjo, Indonesia; ${ }^{3}$ Department of Pharmacology, Faculty of Medicine, Universitas Sebelas Maret, Surakarta, Indonesia
}

Objectives: Depression is a frequent complication of type 2 diabetes mellitus. This study aimed to investigate the relationship between low social support and risk for depression in people with type 2 diabetes through a meta-analysis.

Methods: PubMed, ProQuest, SpringerLink, ScienceDirect, Scopus, the Cochrane Library, Embase, and Google Scholar were searched for English-language articles published up to 2021. Pooled adjusted odds ratios (aORs) were calculated using a random-effect model with $95 \%$ confidence intervals (Cls). Heterogeneity was evaluated by using the Cochrane $Q$ test and $l^{2}$ statistics. The risk of publication bias was estimated using a funnel plot, the Egger test, and the Begg test. The Joanna Briggs Institute Critical Appraisal Tools were used to assess the quality of evidence and the risk of bias.

Results: Eleven studies were included in this meta-analysis, containing a total of 3151 people with type 2 diabetes mellitus. The pooled analysis showed that people with type 2 diabetes mellitus who had low social support had twice as high a risk of depression as those with high social support $(\mathrm{aOR}, 2.02 ; 95 \% \mathrm{Cl}, 1.51$ to $2.70 ; p<0.001)$. A random-effect model was used because the heterogeneity was high $\left(l^{2}=87 \%\right)$.

Conclusions: Low social support was found to increase the risk of depression among people with type 2 diabetes mellitus. Further investigation into factors that may moderate this relationship is required.

Key words: Social support, Depression, Type 2 diabetes mellitus, Meta-analysis

\section{INTRODUCTION}

Type 2 diabetes mellitus (T2DM), which is a metabolic condition involving dysfunctional production and response to insulin or insulin resistance, accounts for around $90 \%$ of all dia-

Received: September 12, 2021 Accepted: October 31, 2021

Corresponding author: Akhmad Azmiardi

Doctoral Program on Public Health, Universitas Sebelas Maret, 36 Ir.

Sutami Street, Surakarta 57126, Indonesia

E-mail: aazmiardi@gmail.com

This is an Open Access article distributed under the terms of the Creative Commons Attribution Non-Commercial License (https://creativecommons.org/licenses/by$\mathrm{nc} / 4.0 / /$ which permits unrestricted non-commercial use, distribution, and reproduction in any medium, provided the original work is properly cited. betes cases [1,2]. T2DM is one of the most common chronic metabolic diseases and constitutes a major health problem affecting the global population [3]. It affected an estimated 462 million people worldwide in 2017, representing $6.28 \%$ of the global population [4]. The prevalence of T2DM is higher in people over the age of 45 and in older adults $[5,6]$.

T2DM is associated with not only short-term complications (e.g., hypoglycemia) and long-term complications (e.g., cardiovascular disease, neuropathy, nephropathy, and retinopathy), but also negative impacts on physical and mental well-being $[7,8]$. Previous studies have shown that depression and anxiety are the most commonly recognized psychological disorders related to T2DM $[9,10]$. 
The prevalence of major depression in people with T2DM is estimated to be around $12 \%$, with reported rates ranging from $8 \%$ to $18 \%$. Milder types of depression, in general, have been reported to be present in 15-35\% of people with T2DM [9]. Previous studies have shown that the prevalence of depression in people with diabetes is twice as high as in those without diabetes. The prevalence of depression in people with T2DM is likely to increase in the future for reasons including the increased life expectancy of patients with diabetes.

Depression is a comorbidity that poses a major challenge for T2DM management [11] because it causes difficulties in starting the treatment and management of patients with diabetes. Depression in people with diabetes hampers diabetes self-care, management practices, and clinical therapy [12]. The coexistence of diabetes and psychological problems also reduces the quality of life $[13,14]$, delays diabetes recovery, and increases the risk of diabetes complications and mortality [15]. A previous study showed that depression was linked with non-compliance to diabetes self-management, including medication adherence, glucose monitoring, physical inactivity, poor dietary habits, sleep disturbance, being prone to live a sedentary lifestyle, and engaging in other risk factors such as smoking and drinking, all of which can lead to poor diabetes control and worse overall clinical outcomes [16].

T2DM impacts nearly every part of individuals' lives, and social networks inevitably play a role in patients' disease [17]. Individuals can use their social networks to satisfy their basic emotional requirements for social integration, increase their selfesteem, and share experiences and information via the interactive process of social support [18]. Social support is a psychosocial element that influences people by providing emotional, informational, companionship, and financial support to increase adherence to diabetes treatment and diabetes management guidelines [19].

In addition, the American Diabetes Association included "support patient behavioral change" as a strategy for improving diabetes care through lifestyle adjustments. People with T2DM must undertake lifestyle adjustments involving social activities, such as healthy eating behavior, exercise, cessation of smoking, weight, and healthy coping [20,21]. Furthermore, previous reviews have shown that a high level of perceived social support leads to improved glycemic control, increased health behaviors, and improved health-related diabetes outcomes [22].

To the best of our knowledge, no studies have systematically explored the association between low social support and depression in people with T2DM. Earlier systematic reviews and meta-analyses were focused on measuring the association between depression and the risk of T2DM incidence [23] and estimating the prevalence of depression among people with T2DM in different regions [24]. Meanwhile, several studies on depression in T2DM are available, but present limited reviews dealing with the impact of low social support and depression. Further systematic evidence is needed on specific measures of social support and depression in people with T2DM.

To improve the health outcomes of people with diabetes living with comorbid depression, it is important to understand the importance of social support in diabetes interventions. This study investigated the relationship between low social support and risk for depression in people with type 2 diabetes through a meta-analysis.

\section{METHODS}

This meta-analysis of observational studies was conducted to assess the association between social support and depression among people with T2DM. This study was carried out according to the PRISMA (Preferred Reporting Items for Systematic Reviews and Meta-Analysis) guidelines.

\section{Eligibility Criteria}

The studies included in this meta-analysis met the following criteria: (1) the study had an observational, cross-sectional design; (2) the predictor of interest was social support and the outcome of interest was depression; (3) the population of the study was people with T2DM; (4) the adjusted odds ratio (aOR) was used in the study as an effect estimate for the association between social support and depression; (5) the full-text article was available in English; and (6) the study received an overall risk of bias score of moderate or lower in the quality assessment, as detailed below. A study was excluded if it only performed bivariate analysis or reported a crude analysis without performing a multivariate analysis as a strategy to deal with confounding factors.

\section{Search Strategy}

A literature search was conducted to find relevant articles from electronic databases up to August 2021 in the English language. The studies identified were from the following databases: PubMed, ProQuest, SpringerLink, ScienceDirect, Scopus, the Cochrane Library, Embase and Google Scholar. The key- 
words used to search the databases were "factors" AND "support" AND "depression" OR "depressive symptom" AND "type 2 diabetes mellitus". These keywords were utilized in combination to search all these databases for relevant literature. In addition, the authors used the internet to search for non-indexed publications and relevant gray literature.

\section{Study Selection}

A screening process was conducted by 2 authors (AA and RPF) independently. In the first stage, titles and abstracts were screened to obtain potential information from relevant studies. Any potential screened studies were then carried forward to the second stage for a full-text review. The full-text articles were assessed and evaluated based on the predefined inclusion criteria. Any differences were resolved by consensus. Resolution from a third reviewer was sought when necessary. Finally, studies that met these criteria were included for data extraction and quality assessment.

\section{Data Extraction}

Two authors (AA and $B M$ ) independently extracted the following data from each study: the author's name, year of publication, the country where the study was performed, study design, settings, range of participants' age, the method of social support assessment, the method of depression assessment, the cut-off values of the instruments, the number of people according to the presence of depression and the degree of social support, the method of diabetes assessment, the aOR and $95 \%$ confidence interval $[\mathrm{Cl}]$, and confounders. Any disagreements about data extraction were settled through consensus.

\section{Quality Assessment}

The quality of the included studies was assessed and rated independently by 2 authors (AA and RPF). It was assessed using the Joanna Briggs Institute (JBI) Critical Appraisal Tools for Use in JBI Systematic Reviews, Checklist for Analytical CrossSectional Studies. This critical appraisal tool had 8 questions, as follows: Q1: Were the criteria for inclusion in the sample clearly defined?; Q2: Were the study subjects and the setting described in detail?; Q3: Was the exposure measured validly and reliably?; Q4: Were objective standard criteria used for measurement of the condition; Q5 Were confounding factors identified?; Q6: Were strategies to deal with confounding factors stated?; Q7: Were the outcomes measured validly and reli- ably?; and Q8: Was appropriate statistical analysis used?. Each question could be rated in 4 categories: yes, no, unclear, and not applicable. A study was considered as having a high risk of bias if $\leq 49 \%$ of the answers were positive, a moderate risk of bias if between $50 \%$ and $69 \%$ of the questions had positive answers, and a low risk of bias if $\geq 70 \%$ of the answers were positive. Any disagreements about data extraction were settled through consensus.

\section{Statistical Analysis}

The study results included in the analysis were summarized and tabulated. All included studies expressed odds ratios (ORs) as the measure of effect on depression. The OR is commonly used to report meta-analysis results if the results are binary, which is compatible with the included studies. The estimated association between social support and depression was evaluated by using aORs and $95 \%$ Cls. Review Manager (RevMan) 5.3 (Cochrane, London, UK) and Stata version 16.0 (StataCorp., College Station, TX, USA) were used to analyze the extracted data. The results of the meta-analysis were summarized in a forest plot, and a $p$-value of less than 0.05 was considered to indicate statistical significance. The heterogeneity of the included studies was evaluated using the Cochrane $Q$ test and $l^{2}$ statistics. A Cochrane $Q$ test ( $p$-value) less than 0.10 indicated significant heterogeneity. Meanwhile, $l^{2}$ values less than $25 \%$ indicated mild heterogeneity, values between $25 \%$ and $50 \%$ indicated moderate heterogeneity, and values greater than $50 \%$ indicated significant heterogeneity among studies. A random-effects model was used if the data were heterogeneous. Potential publication bias was assessed by using funnel plots, the Egger test, and the Begg statistical test. Funnel plots were used to visually detect publication bias. The presence of an asymmetric funnel plot indicated that publication bias was present; otherwise, a symmetrical funnel plot indicated no publication bias. The Egger and Begg statistical tests were used to check the statistical significance of publication bias, with $p$-values less than 0.05 indicating potential publication bias. A sensitivity analysis was conducted to investigate the effect of each study on the pooled OR by sequentially excluding each study.

\section{Ethics Statement}

This article does not contain any studies with human participants performed by any of the authors. 


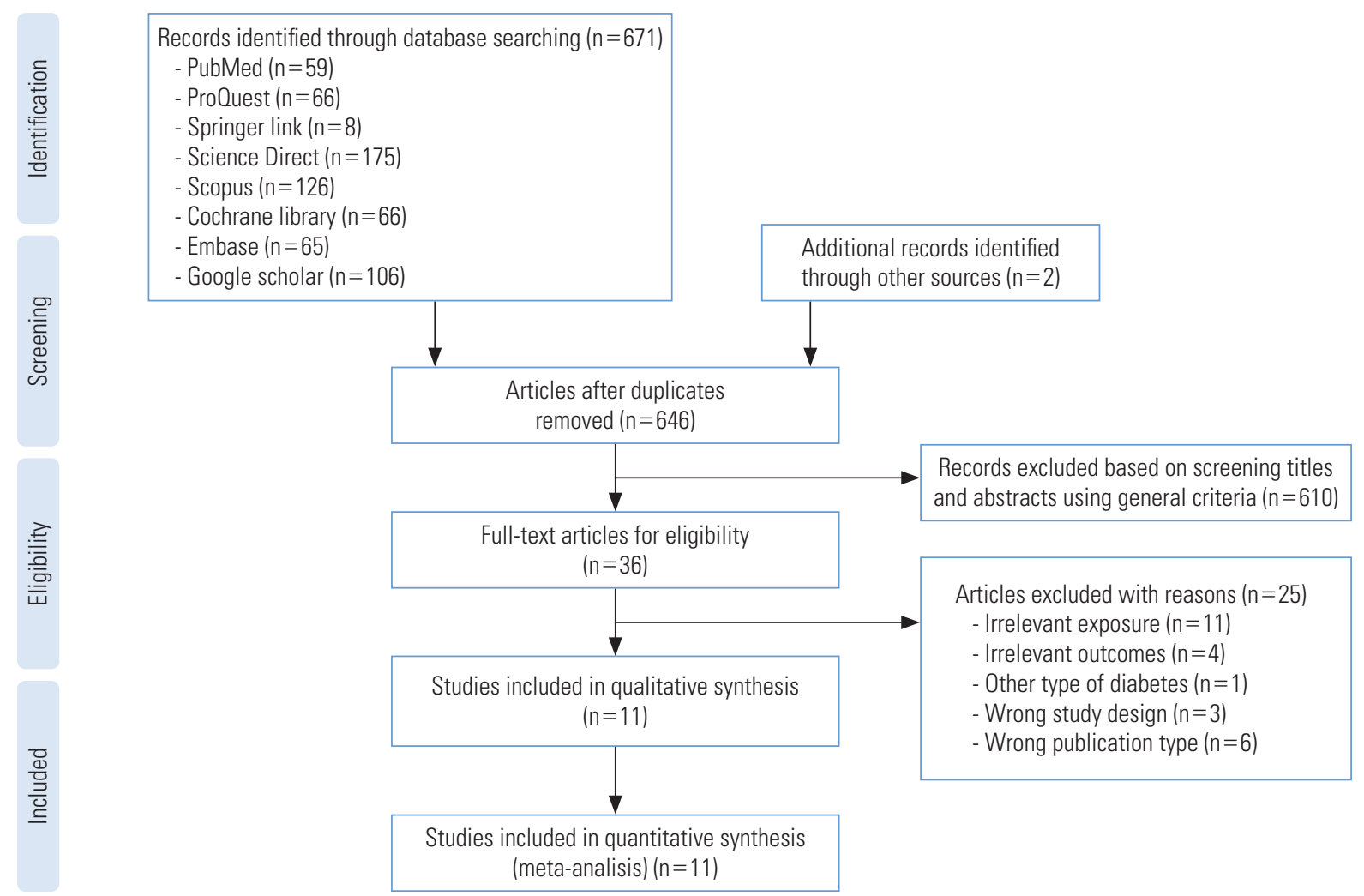

Figure 1. PRISMA (Preferred Reporting Items for System atic Reviews and Meta-Analysis) flowchart.

\section{RESULTS}

\section{Study Selection}

A PRISMA flow diagram of study inclusion is presented in Figure 1. The database search resulted in 671 records from the 8 sources. Two records were also generated from non-indexed publications and the relevant gray literature using web search engines. All records were published up to August 2021. Twentyfive duplicates were removed. Initial screening based on the title and abstract using general criteria identified 36 records that were potentially eligible articles. A full-text review of the studies was then conducted, and the studies were assessed according to the inclusion criteria. Twenty-five articles were excluded due to various reasons such as irrelevant exposure, irrelevant outcomes, other types of diabetes, different study design, and different publication types. Finally, 11 articles satisfied the inclusion criteria and were included in the systematic review and meta-analysis.

\section{Characteristics of Included Studies}

Table 1 describes the characteristics of the studies included in the meta-analysis, which consisted of the author, year of publication, country, study design, setting, age range, method of social support assessment, method of depression assessment, the cut-off score of the instrument, number of people according to the presence of depression and the degree of social support, method of diabetes assessment, aOR and $95 \% \mathrm{Cl}$, and confounders. The 11 articles were published up to August 2021 and involved a total of 3156 participants. Two studies were conducted in the United States [25,26], 2 in Saudi Arabia [27,28], 6 in Africa (5 in Ethiopia and 1 in Nigeria) [29-34], and 1 in Thailand [35]. All included studies were cross-sectional. Among the included studies, 9 were conducted in a hospitalbased setting $[26-33,35]$ and 2 were in a community-based setting [25]. The sample size of the studies ranged from 200 to 403. The Center for Epidemiologic Studies-Depression (CES-D) scale $(n=3)$ [25-27], Patient Health Questionnaire-9 (PHQ-9) $(n=3)$ [29-31], and Hospital Anxiety and Depression Scale (HADS) $(n=3)[28,32,35]$ were most often used to measure depression. The Beck Depression Inventory (BDI) $(n=1)$ [34] and the Zung Self Depression Rating Scale $(n=1)$ [33] were used in the remaining studies. Social support was assessed by various questionnaires including the Oslo Social Support Scale $(n=2)$ [30,32], Perceived Social Support Scale (PSSS) $(n=2)[27,33]$, 


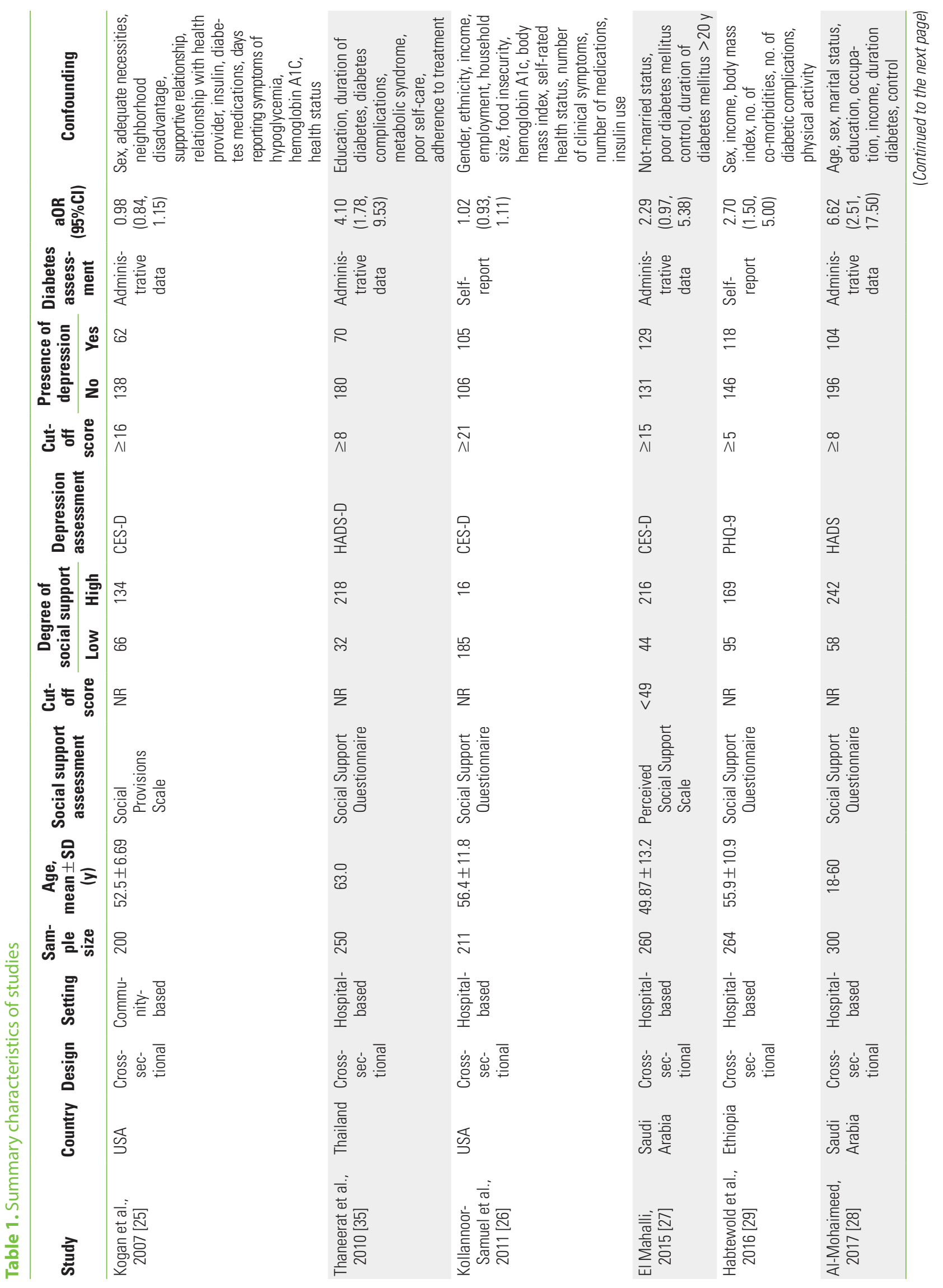




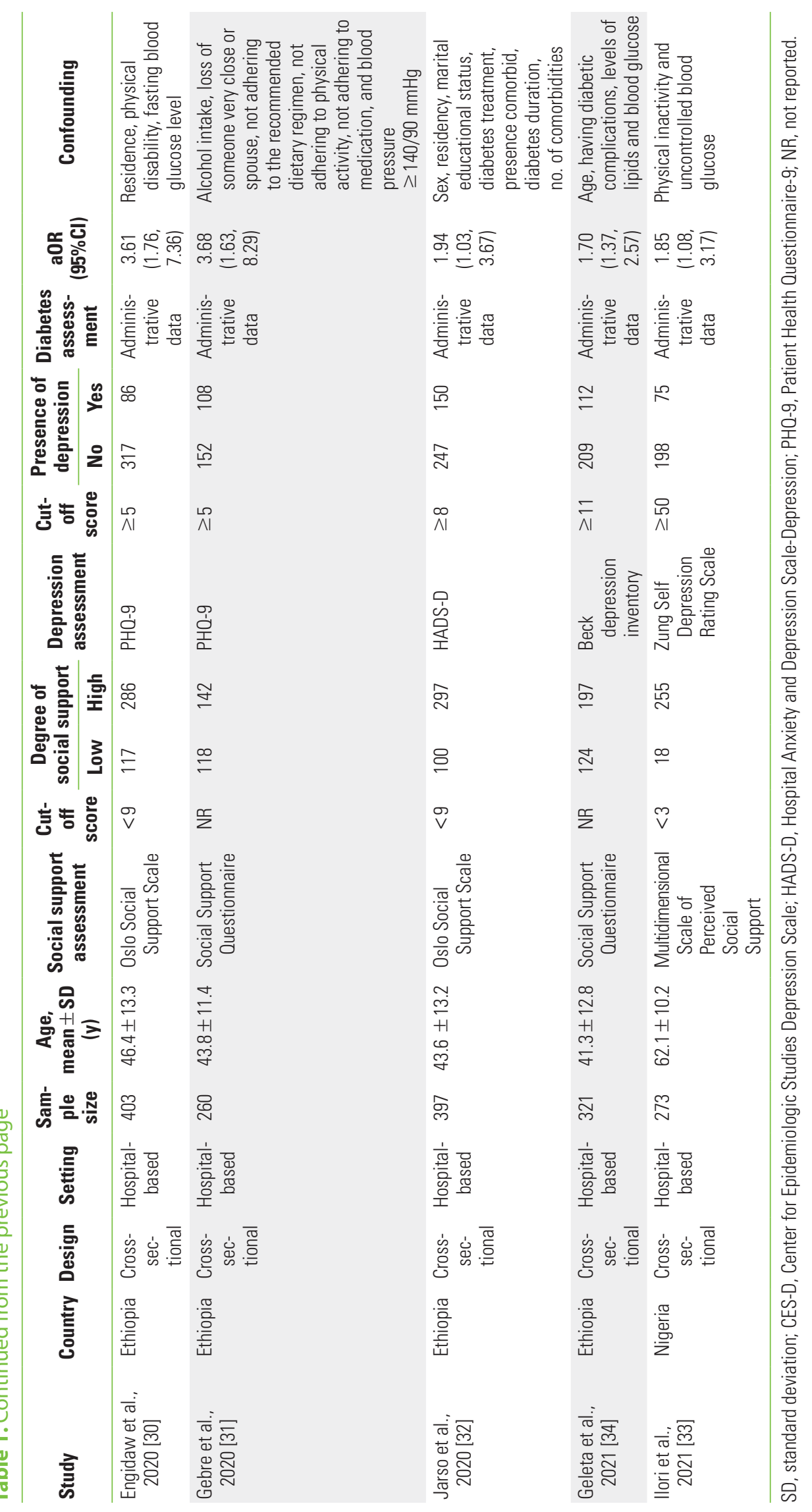


Social Provision Scale $(n=1)$ [25], and other validated questionnaires $(n=6)[26,28,29,31,34,35]$. T2DM was assessed using the self-report of physician-diagnosed diabetes $(n=2)$ and administrative data or medical records $(n=9)$. A detailed description of the study characteristics, including the cut-off value of the instruments, is summarized in Table 1.

\section{Risk of Bias}

All the studies considered had a low risk of bias with a high percentage of positive responses to the JBI critical appraisal tools for cross-sectional studies. Only 3 studies did not sufficiently report the inclusion and exclusion criteria. The risk of bias is summarized in detail in Table 2 .

\section{Meta-Analysis Results}

Figure 2 presents a forest plot showing the OR and $95 \% \mathrm{Cl}$ of each study and the pooled aOR. The pooled analysis showed an association between social support and depression, in which low social support significantly increased the risk of depression among people with T2DM (OR, 2.02; 95\% Cl, 1.51 to $2.70 ; p<$

Table 2. Risk of bias assessed by the Joanna Briggs Institute (JBI) Critical Appraisal Tools for Use in JBI Systematic Reviews, Checklist for Analytical Cross-Sectional Studies

\begin{tabular}{|c|c|c|c|c|c|c|c|c|c|c|}
\hline Study & 01 & 02 & 03 & 04 & 05 & 06 & 07 & 08 & $\%$ Yes & Risk \\
\hline Kogan et al., 2007 [25] & Yes & Yes & Yes & Yes & Yes & Yes & Yes & Yes & 100 & Low \\
\hline Thaneerat et al., 2010 [35] & Yes & Yes & Yes & Yes & Yes & Yes & Yes & Yes & 100 & Low \\
\hline El Mahalli, 2015 [27] & Yes & Yes & Yes & Yes & Yes & Yes & Yes & Yes & 100 & Low \\
\hline Habtewold et al., 2016 [29] & Yes & Yes & Yes & Yes & Yes & Yes & Yes & yes & 100 & Low \\
\hline Gebre et al., 2020 [31] & Unclear & Yes & Yes & Yes & yes & Yes & Yes & Yes & 87.5 & Low \\
\hline Jarso et al., 2020 [32] & Unclear & Yes & Yes & Yes & Yes & Yes & Yes & Yes & 87.5 & Low \\
\hline Geleta et al., 2021 [34] & Yes & Yes & Yes & Yes & Yes & Yes & Yes & yes & 100 & Low \\
\hline Ilori et al., 2021 [33] & Unclear & Yes & Yes & Yes & Yes & Yes & Yes & Yes & 87.5 & Low \\
\hline
\end{tabular}

01: Were the criteria for inclusion in the sample clearly defined?; 02: Were the study subjects and the setting described in detail?; 03: Was the exposure measured in a valid and reliable way?; 04: Were objective, standard criteria used for measurement of the condition?; 05: Were confounding factors identified?; 06: Were strategies to deal with confounding factors stated?; 07: Were the outcomes measured in a valid and reliable way?; and 08: Was appropriate statistical analysis used?

OR

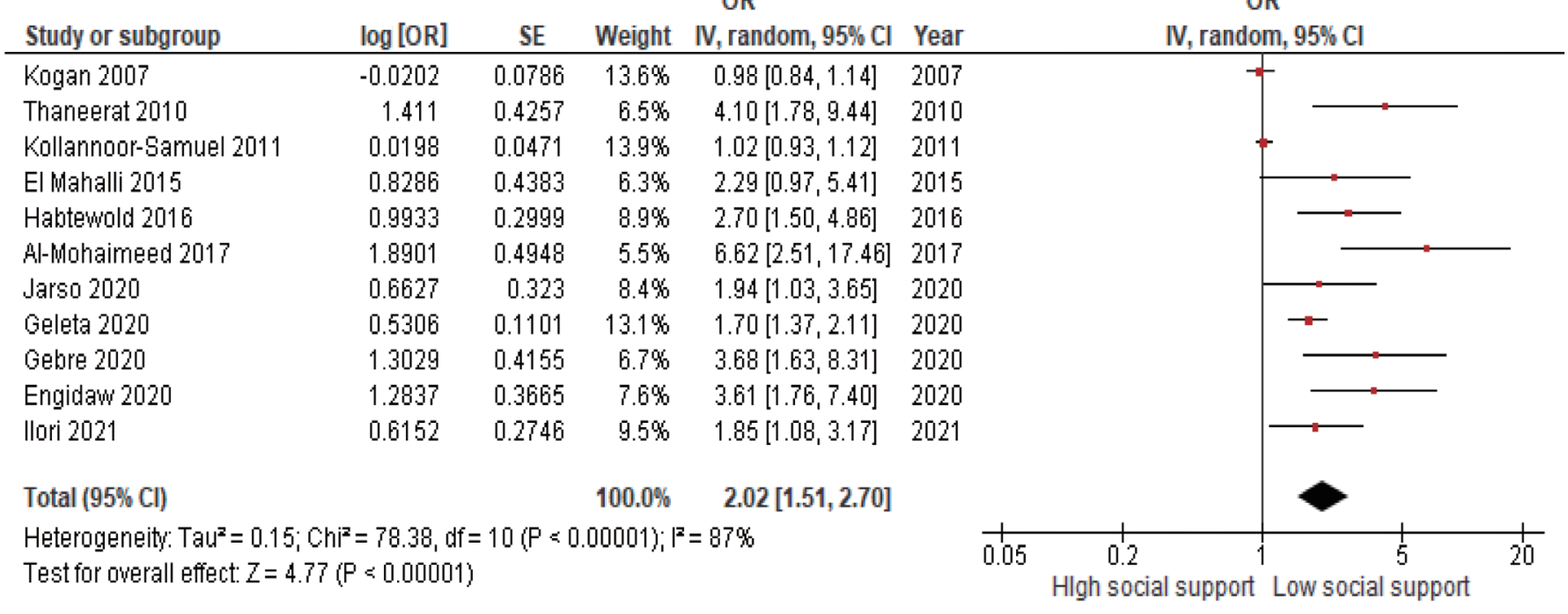

Figure 2. Forest plot of the effect of social support on the depression in studies of type 2 diabetes mellitus. OR, odds ratio; SE, standard error; $\mathrm{Cl}$, confidence interval. 


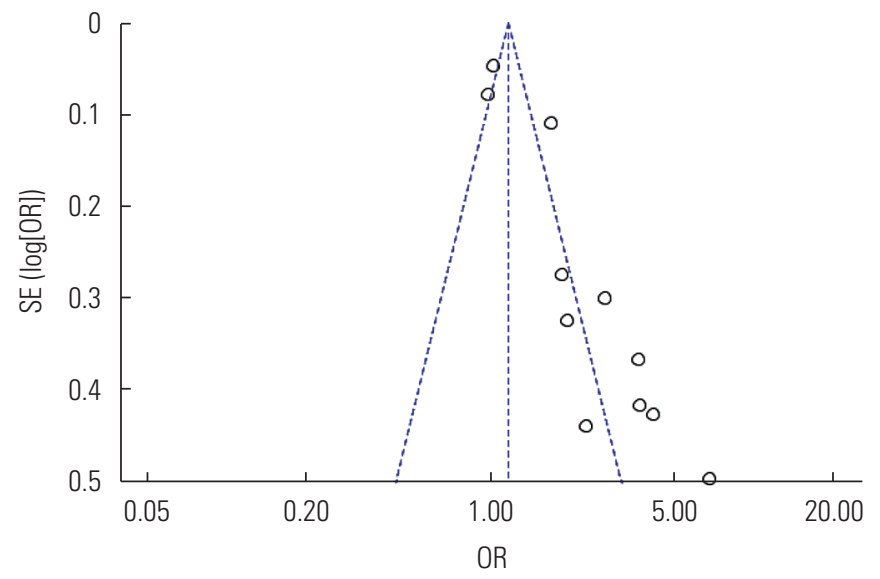

Figure 3. Funnel plot of publication bias for depression in studies of type 2 diabetes mellitus. SE, standard error; OR, odds ratio.

0.001). The heterogeneity among studies was high and statistically significant $\left(l^{2}=87 \%, p<0.001\right)$, so a random-effect model was adopted. Publication bias in the studies was evaluated using a funnel plot, the Egger test, and the Begg test. The funnel plot showed an asymmetrical distribution that indicated a possibility of publication bias (Figure 3). This was also supported by the results of the Egger and Begg tests, which indicated a possibility of publication bias ( $p=0.044$ and $p<0.001$, respectively). A sensitivity analysis was performed to see whether any single study had an impact on the overall meta-analysis result using a random-effects model. Each study was sequentially excluded to test the robustness of the pooled aOR of depression. The findings revealed that there was no strong evidence that a single study had an impact on the overall result of the meta-analysis, since the single study estimates were closer to the combined estimate (Table 3 ).

\section{DISCUSSION}

The present systematic review and meta-analysis comprised 11 studies containing a total of 3156 people with T2DM. All included studies were cross-sectional. The main goal of this study was to evaluate the relationship between low social support and risk for depression in people with T2DM. According to the meta-analysis results, social support was significantly associated with depression. People with T2DM who had low social support had twice as high a risk of depression as those with high social support. People with T2DM who have low social support may be more prone to develop depression. The statis-
Table 3. Sensitivity analysis of the effects of single studies on the overall findings for the relationship between social support and depression in patients with type 2 diabetes mellitus

\begin{tabular}{lcccc}
\hline \multirow{2}{*}{ Study } & \multicolumn{2}{c}{$\begin{array}{c}\text { Sample } \\
\text { size }\end{array}$} & $\begin{array}{c}\text { Pooled } \\
\text { a0R }\end{array}$ & \multicolumn{2}{c}{ 95\% CI } \\
\hline Kogan et al., 2007 [25] & 200 & 2.37 & 1.62 & UL \\
\hline Thaneerat et al., 2010 [35] & 250 & 1.90 & 1.43 & 2.54 \\
Kollannoor-Samuel et al., 2011 [26] & 211 & 2.36 & 1.62 & 3.43 \\
El Mahalli, 2015 [27] & 260 & 2.00 & 1.49 & 2.70 \\
Habtewold et al., 2016 [29] & 276 & 1.95 & 1.45 & 2.62 \\
Al-Mohaimeed, 2017 [28] & 300 & 1.86 & 1.40 & 2.46 \\
Engidaw et al., 2020 [30] & 403 & 1.90 & 1.43 & 2.54 \\
Gebre et al., 2020 [31] & 260 & 1.92 & 1.44 & 2.56 \\
Jarso et al., 2020 [32] & 397 & 2.03 & 1.50 & 2.75 \\
Geleta et al., 2021 [34] & 321 & 2.09 & 1.52 & 2.88 \\
Ilori et al., 2021 [33] & 273 & 2.04 & 1.51 & 2.77 \\
Combined & 3151 & 2.02 & 1.51 & 2.70 \\
\hline
\end{tabular}

aOR, adjusted odds ratio; Cl, confidence interval; LL, lower limit; UL, upper limit.

tical test for publication bias was significant. Each included study had a low risk of bias, so the quality of the studies was good. There was high heterogeneity among studies, which may have been due to the wide range of depression scales used, the various methods of social support assessment, and diversity in baseline characteristics such as the age range and setting.

To the best of our knowledge, this is the first meta-analysis examining social support and depression. The present study showed that the prevalence of depression in people with T2DM was high in the included studies. This finding is in line with a prior meta-analysis showing that depression was common among T2DM patients in India. Depression was more frequently related to the presence of problems in persons who have T2DM [24]. In order to prevent these problems, social support is needed. Social support improves mental health and quality of life by helping people feel valued and connected to their social networks. This sense of belonging is connected to fewer mental health difficulties and, thus, serves as a strategy to prevent depression [36].

According to the previous systematic review, social support is an important protective factor against depression. Sources of social support can vary, including support from parents, spouses, and friends. These results suggest that social support be a major target for reducing and preventing depression and its consequences [37]. This finding is in line with those of previous studies showing that social support is a protective factor against depression $[38,39]$. 
Depression is a prevalent mental illness in people with diabetes [11]. Depression frequently manifests as cognitive impairment with insufficient coping skills in the psychological and social domains. Older individuals may lose interest in physical and social activities, increasing their risk of impaired physical function and falls. Depression in people with diabetes is linked to several factors, including low socioeconomic status, the presence of diabetic complications, the presence of medical comorbidity, lower education, poor glycemic control, family history of depression, female sex, younger age, not having a spouse, and poor social support [9].

Social support is becoming increasingly essential in the management of people with T2DM. It has been associated with the development of problems related to diabetes [40], as well as psychological well-being [41]. When patients perceive social support from others, they feel cared about, accepted, and appreciated and their subjective well-being rises; as a result, they adopt a positive attitude toward their chronic diseases [42]. Spouses, family, and friends, in general, provide social support and affect health-related decision-making in individuals with T2DM. Individuals who receive support will have greater psychological ease and be more able to deal with health issues [43].

A study by loannou et al. [44] showed that social support was a protective factor against depression. In other words, people with higher social support had a lower risk of depression than those with lower social support. People with high levels of stress may be less able to maintain close social contact and relationships with others. The absence of social connections in people with depression deprives them of the opportunity to attain a level of perceived significance that would enable them to deal with depression. It is also possible that people with depression are unable to use social support in a way that maximizes its benefits for their treatment, psychological well-being, and quality of life. This finding supports previous studies that found social support to have a significant effect on diabetes self-care and quality of life $[22,45]$.

Furthermore, a study by Ekem-Ferguson et al. [46] about the influence of perceived social support on depression among T2DM patients indicated that a supportive atmosphere is a source of social support that helps improve patients' mental condition. Members of patients' support networks give basic information on illnesses, self-care or management techniques, and strategies to handle problems. They also interact with their peers and share their experiences and best practices in managing diabetes. Social support can also help patients develop coping skills and structure in their daily routines, helping them to cope with stressful situations, stick to their treatment plan through tough times, and reduce the risk of stress-related disease [47].

In addition, if left untreated, depression can have significant negative consequences for the health and physical and mental well-being of patients with T2DM. Healthy coping with depression by involving social support in treatment may help remove psychological obstacles to adherence with diabetes selfcare, which is the ultimate goal of diabetes management. Gaining a better understanding of the role of social support and depression in T2DM will contribute to our understanding of this issue and enable practitioners to design effective social support-based interventions.

Family-based and peer-based interventions are strategies associated with diabetes self-management support [48]. Technology is a promising method to help family and friends provide social support. For example, by utilizing an online health community that provides social support and consultation with doctors. Not only the patient, but also the patient's family, can use the online health communities as a simple way to find others who have been in similar circumstances to share knowledge and experiences, and obtain personal stories and tips. In an online social environment, users may grow their social network and interact with others at any time, regardless of socioeconomic status. In contrast, it is difficult to find as many peers to interact with in an offline setting [49].

This study has several strengths, including the provision of a novel perspective on the association between social support and depression. We conducted a comprehensive assessment of the literature with no time constraints, including both published and unpublished research from several online databases. A wide search strategy was employed to gather all relevant studies. The included studies were those deemed to have a low risk of bias as defined using the JBI Critical Appraisal Tools for Use in JBI Systematic Reviews, Checklist for Analytical CrossSectional Studies. Furthermore, several sensitivity analyses were conducted to assess potential sources of heterogeneity in our results.

Nonetheless, there are several limitations in this systematic review and meta-analysis. First, self-report questionnaires were used to measure depression in the included studies, but different questionnaires with different cut-off values were used to identify cases of depression from study to study. If a self-reported questionnaire has a lower cut-off value, the incidence of 
clinically relevant levels of depression in people with diabetes may have been overestimated.

Second, there was high heterogeneity among included studies $\left(I^{2}=87 \%\right)$. Heterogeneity may have also arisen from differences in characteristics such as the study setting, the age of the participants, methods of depression assessment, and methods of social support assessment. Third, the included studies were limited to English-language articles. Although the statistical analysis did not detect potential publication bias, there is nonetheless potential publication bias due to language restrictions. Finally, all the studies examined in this review had cross-sectional study designs; as a result, the outcome variable may have been influenced by other confounding variables. This might have reduced the study's power and the likelihood of a causal relationship between social support and depression in people with T2DM.

\section{CONCLUSION}

This meta-analysis has shown an association between social support and depression among people with T2DM. The findings suggest that diabetes treatment programs should include screening for depression early in people with T2DM. Healthcare professionals, family, spouses, and friends must provide support through proper diabetes management education programs. The nature of the association between social support and depression remains unknown, and future studies should explore the role of unidentified possible mediators or moderators in this relationship. Nevertheless, meta-analysis is a useful tool for assessing the consistency of study results and can give insights and suggestions for future research.

\section{CONFLICT OF INTEREST}

The authors have no conflicts of interest associated with the material presented in this paper.

\section{FUNDING}

None.

\section{ACKNOWLEDGEMENTS}

We thank the authors of the studies included in this metaanalysis.

\section{AUTHOR CONTRIBUTIONS}

Conceptualization: Azmiardi A, Murti B, Febrinasari RP. Data curation: Azmiardi A, Murti B., Formal analysis: Azmiardi A, Murti B. Funding acquisition: None. Methodology: Azmiardi A, Murti B. Project administration: Azmiardi A, Murti B, Febrinasari RP, Tamtomo DG. Visualization: Azmiardi A. Writing - original draft: Azmiardi A, Murti B, Febrinasari RP, Tamtomo DG. Writing - review \& editing: Azmiardi A, Murti B, Febrinasari RP.

\section{ORCID}

Akhmad Azmiardi $\quad$ https://orcid.org/0000-0002-7021-4528
Bhisma Murti $\quad$ https://orcid.org/0000-0001-9405-3872
$\begin{gathered}\text { Ratih Puspita Febrinasari } \\ \text { https://orcid.org/0000-0001-9767-3284 }\end{gathered}$

Didik Gunawan Tamtomo https://orcid.org/0000-0002-2005-2444

\section{REFERENCES}

1. American Diabetes Association. 2. Classification and diagnosis of diabetes: standards of medical care in diabetes-2018. Diabetes Care 2018;41(Suppl 1):S13-S27.

2. Galicia-Garcia U, Benito-Vicente A, Jebari S, Larrea-Sebal A, Siddiqi $\mathrm{H}$, Uribe KB, et al. Pathophysiology of type 2 diabetes mellitus. Int J Mol Sci 2020;21(17):6275.

3. Saeedi P, Petersohn I, Salpea P, Malanda B, Karuranga S, Unwin $\mathrm{N}$, et al. Global and regional diabetes prevalence estimates for 2019 and projections for 2030 and 2045: results from the International Diabetes Federation Diabetes Atlas, 9th edition. Diabetes Res Clin Pract 2019;157:107843.

4. Khan MA, Hashim MJ, King JK, Govender RD, Mustafa H, Al Kaabi J. Epidemiology of type 2 diabetes - global burden of disease and forecasted trends. J Epidemiol Glob Health 2020; 10(1):107-111.

5. Introduction: standards of medical care in diabetes-2018. Diabetes Care 2018;41(Suppl 1):S1-S2.

6. Ang GY. Age of onset of diabetes and all-cause mortality. World J Diabetes 2020;11(4):95-99.

7. Kalra S, Jena BN, Yeravdekar R. Emotional and psychological needs of people with diabetes. Indian J Endocrinol Metab 2018;22(5):696-704.

8. Alzoubi A, Abunaser R, Khassawneh A, Alfaqih M, Khasawneh $A$, Abdo N. The bidirectional relationship between diabetes 
and depression: a literature review. Korean J Fam Med 2018; 39(3):137-146.

9. Andreoulakis E, Hyphantis T, Kandylis D, lacovides A. Depression in diabetes mellitus: a comprehensive review. Hippokratia 2012;16(3):205-214.

10. Sharma K, Dhungana G, Adhikari S, Bista Pandey A, Sharma M. Depression and anxiety among patients with type ii diabetes mellitus in Chitwan Medical College Teaching Hospital, Nepal. Nurs Res Pract 2021;2021:8846915.

11. Sartorius N. Depression and diabetes. Dialogues Clin Neurosci 2018;20(1):47-52.

12. Owens-Gary MD, Zhang X, Jawanda S, Bullard KM, Allweiss $P$, Smith BD. The importance of addressing depression and diabetes distress in adults with type 2 diabetes. J Gen Intern Med 2019;34(2):320-324.

13. Lee HW, Song M, Yang JJ, Kang D. Determinants of poor selfrated health in Korean adults with diabetes. J Prev Med Public Health 2015;48(6):287-300.

14. Bahety P, Agarwal G, Khandelwal D, Dutta D, Kalra S, Taparia P, et al. Occurrence and predictors of depression and poor quality of life among patients with type-2 diabetes: a Northern India perspective. Indian J Endocrinol Metab 2017;21(4):564569.

15. Wu CS, Hsu LY, Wang SH. Association of depression and diabetes complications and mortality: a population-based cohort study. Epidemiol Psychiatr Sci 2020;29:e96.

16. Jeon EJ. Diabetes and depression. Yeungnam Univ J Med 2018; 35(1):27-35.

17. Schram MT, Assendelft WJ, van Tilburg TG, Dukers-Muijrers $\mathrm{NH}$. Social networks and type 2 diabetes: a narrative review. Diabetologia 2021;64(9):1905-1916.

18. Tang F, Chi I, Dong X. The relationship of social engagement and social support with sense of community. J Gerontol A Biol Sci Med Sci 2017;72(suppl_1):S102-S107.

19. Strom JL, Egede LE. The impact of social support on outcomes in adult patients with type 2 diabetes: a systematic review. Curr Diab Rep 2012;12(6):769-781.

20. Sevild CH, Niemiec CP, Bru LE, Dyrstad SM, Husebø AM. Initiation and maintenance of lifestyle changes among participants in a healthy life centre: a qualitative study. BMC Public Health 2020;20(1):1006.

21. American Diabetes Association. Standards of medical care in diabetes-2016 abridged for primary care providers. Clin Diabetes 2016;34(1):3-21.

22. Rad GS, Bakht LA, Feizi A, Mohebi S. Importance of social sup- port in diabetes care. J Educ Health Promot 2013;2:62.

23. Graham EA, Deschênes SS, Khalil MN, Danna S, Filion KB, Schmitz $\mathrm{N}$. Measures of depression and risk of type 2 diabetes: a systematic review and meta-analysis. J Affect Disord 2020;265: 224-232.

24. Hussain S, Habib A, Singh A, Akhtar M, Najmi AK. Prevalence of depression among type 2 diabetes mellitus patients in India: a meta-analysis. Psychiatry Res 2018;270:264-273.

25. Kogan SM, Brody GH, Crawley C, Logan P, Murry VM. Correlates of elevated depressive symptoms among rural African American adults with type 2 diabetes. Ethn Dis 2007;17(1):106-112.

26. Kollannoor-Samuel G, Wagner J, Damio G, Segura-Pérez S, Chhabra J, Vega-López S, et al. Social support modifies the association between household food insecurity and depression among Latinos with uncontrolled type 2 diabetes. J Immigr Minor Health 2011;13(6):982-989.

27. El Mahalli AA. Prevalence and predictors of depression among type 2 diabetes mellitus outpatients in Eastern Province, Saudi Arabia. Int J Health Sci (Qassim) 2015;9(2):119-126.

28. Al-Mohaimeed AA. Prevalence and factors associated with anxiety and depression among type 2 diabetes in Qassim: a descriptive cross-sectional study. J Taibah Univ Med Sci 2017; 12(5):430-436.

29. Habtewold TD, Alemu SM, Haile YG. Sociodemographic, clinical, and psychosocial factors associated with depression among type 2 diabetic outpatients in Black Lion General Specialized Hospital, Addis Ababa, Ethiopia: a cross-sectional study. BMC Psychiatry 2016;16:103.

30. Engidaw NA, Wubetu AD, Basha EA. Prevalence of depression and its associated factors among patients with diabetes mellitus at Tirunesh-Beijing general hospital, Addis Ababa, Ethiopia. BMC Public Health 2020;20(1):266.

31. Gebre BB, Anand S, Assefa ZM. Depression and its predictors among diabetes mellitus patients attending treatment in Hawassa University Comprehensive Specialized Hospital, southern Ethiopia. J Diabetes Res 2020 Jan 22;2020:7138513.

32. Jarso MH, Likasa DD. Prevalence and associated factors of depression among diabetic outpatients in Ethiopia. Prim Care Companion CNS Disord 2020;22(2):19m02479.

33. Ilori HT, Salawu AT, Fawole OI. Depression and anxiety among patients with type 2 diabetes mellitus in Ibadan, Oyo State. Afr J Med Med Sci 2021;50(2):189-197.

34. Geleta BA, Dingata ST, Emanu MD, Kebede EB, Eba LB, Abera $\mathrm{KB}$. Prevalence of depression and associated factors among type 2 diabetes patients attending hospitals in llu AbaBor and 
Bunno Bedelle Zones, South West Ethiopia, 2020: a cross sectional study. J Depress Anxiety 2021;9:388.

35. Thaneerat T, Tangwongchai S, Worakul P. Prevalence of depression, hemoglobin A1C level, and associated factors in outpatients with type-2 diabetes. Asian Biomed 2010;3(4):383-390.

36. Camara M, Bacigalupe G, Padilla P. The role of social support in adolescents: are you helping me or stressing me out? Int J Adolesc Youth 2017;22(2):123-136.

37. Schmitz N, Gariépy G, Smith KJ, Malla A, Wang J, Boyer R, et al. The pattern of depressive symptoms in people with type 2 diabetes: a prospective community study. J Psychosom Res 2013; 74(2):128-134.

38. Kaya UP, Caydam OD. Association between social support and diabetes burden among elderly patients with diabetes: a crosssectional study from Turkey. Saudi J Med Med Sci 2019;7(2): 86-92.

39. Beverly EA, Ritholz MD, Dhanyamraju K. The buffering effect of social support on diabetes distress and depressive symptoms in adults with type 1 and type 2 diabetes. Diabet Med 2021;38(4):e14472.

40. Li X, Zhang S, Xu H, Tang X, Zhou H, Yuan J, et al. Type D personality predicts poor medication adherence in Chinese patients with type 2 diabetes mellitus: a six-month follow-up study. PLoS One 2016;11(2):e0146892.

41. Mojahed A, Fallah M, Ganjali A, Heidari A. The role of social support and coping strategies in prediction of psychological well being in type 2 diabetic patients of Zahedan. Bali Med J 2019;8(1):281-286.

42. Cobo-Rendón R, López-Angulo Y, Pérez-Villalobos MV, Díaz-
Mujica A. Perceived social support and its effects on changes in the affective and eudaimonic well-being of Chilean university students. Front Psychol 2020;11:590513.

43. Ozbay F, Johnson DC, Dimoulas E, Morgan CA, Charney D, Southwick S. Social support and resilience to stress: from neurobiology to clinical practice. Psychiatry (Edgmont) 2007;4(5):35-40.

44. loannou M, Kassianos AP, Symeou M. Coping with depressive symptoms in young adults: perceived social support protects against depressive symptoms only under moderate levels of stress. Front Psychol 2019;9:2780.

45. Liu Y, Maier M, Hao Y, Chen Y, Qin Y, Huo R. Factors related to quality of life for patients with type 2 diabetes with or without depressive symptoms-results from a community-based study in China. J Clin Nurs 2013;22(1-2):80-88.

46. Ekem-Ferguson G, Tetteh J, Udofia EA, Doku A, Swaray SM, Mohammed $S$, et al. Influence of perceived social support on depression among type 2 diabetic patients: a concurrent crosssectional mixed-method study. Health Sci Investig J 2020;1(2): 93-104.

47. Ramkisson S, Pillay BJ, Sibanda W. Social support and coping in adults with type 2 diabetes. Afr J Prim Health Care Fam Med 20171;9(1):e1-e8.

48. Leggatt M, Woodhead G. Family peer support work in an early intervention youth mental health service. Early Interv Psychiatry 2016;10(5):446-451.

49. Li Y, Yan X. How could peers in online health community help improve health behavior. Int J Environ Res Public Health 2020; 17(9):2995. 